\title{
LA MODERNIZACIÓN DE LA GESTIÓN PÚBLICA EN EL PERÚ
}

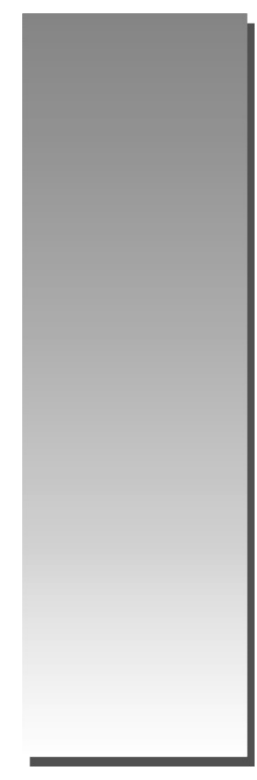

\section{Luis Fernando Valeriano Ortíz* Ifernandovaleriano@yahoo.es}

\section{RESUMEN}

La modernización de la gestión pública en el Perú es uno de los pilares más importantes para el desarrollo sostenible del país, se requiere de una voluntad política consensuada entre los poderes del Estado, de un marco legal que comprenda al gobierno central, regional y local en forma integral dentro de una concepción sistémica, que tome en cuenta las experiencias comprendidas en los logros alcanzados y sus aspectos restrictivos, con respecto a la jurisprudencia de leyes sobre la reforma de la administración pública del país, que fueron promulgadas e implementadas por los diversos gobiernos en estas últimas décadas y cuyos resultados no fueron satisfactorios en términos generales.

Esta breve caracterización de la problemática requiere de un mayor análisis, que servirá de base para proyectar un dispositivo legal, que por la naturaleza de su contenido, requiere ser ampliamente debatido en las diversas instancias de los poderes del Estado para su aprobación, además deberá responder a las exigencias de una gestión pública de excelencia, que contribuya a mejorar la calidad de los servicios que se presta a la sociedad y a una revalorización del trabajador del sector público. Para tal efecto es importante revisar los antecedentes de reformas de la administración pública, que se han desarrollado en países que han tenido éxito y tomar en cuenta su incidencia en el desarrollo de la regionalización de los sectores productivos y de servicios, con el propósito de conocer las fortalezas y debilidades del frente interno nacional y las oportunidades y amenazas del entorno y en este contexto plantear una Reforma de la Administración Pública, que tome en cuenta las tecnologías de las informaciones y de las comunicaciones electrónica.

* Doctor en administración, Licenciado en administración, docente permanente de pregrado y postgrado de la Facultad de Ciencias Administrativas de la UNMSM, especialista en Proyectos de Inversión Pública. 


\section{ABSTRACT}

Modernizing Governance in Peru is one of the most important pillars for the country's sustainable development, requires a political consensus between the branches of government, a legal framework, which includes the central government, regional and Local integrally within a systemic concept that takes into account the experiences included in the achievements and restrictive aspects, regarding the case of laws on public administration reform in the country, which were promulgated and implemented by the various governments in recent decades, and the results were not satisfactory overall.

This brief description of the problem, requires further analysis as a basis for projecting a legal device, which by the nature of its content, needs to be widely discussed in the various bodies of state authorities for approval, and must meet the needs of public management of excellence, to help improve the quality of services provided to society and appreciation of public sector workers, for this purpose it is important to review the history of the Public Administration Reform, that have been developed in countries that have been successful and take into account their impact on the development of the regionalization of production and service sectors, in order to know the strengths and weaknesses of the national home front and the opportunities and threats in the environment in this context and propose a reform of the public administration, which takes into account the information technologies and electronic communications.

\section{INTRODUCCIÓN}

Es importante precisar, que el crecimiento de la macroeconomía del país, en estas últimas décadas, basada en una economía de mercado, se ve reflejado en los factores, variables e indicadores de la macroeconomía nacional, difundidas en los boletines del Ministerio de Economía y Finanzas, del Banco Central de Reserva y del Instituto Nacional de Estadística y Censo a nivel de Perú, también recogidas en los informes económicos del Fondo Monetario Internacional, Banco Mundial, Banco Interamericano de Desarrollo, etc. (organismos multinacionales) que refuerzan la confianza del Perú, para promover y generar un clima de seguridad para el inversionista nacional y extranjero, para ello se requiere de un sector público nacional eficiente, eficaz y efectivo al servicio de la sociedad peruana, lejos de modelos burocráticos, organizaciones paquidérmicas y de economías planificadas en proceso de extinción.

Mientras que países desarrollados como Estados Unidos de Norteamérica y los países de Europa del sur, como Francia, Italia, Grecia, Portugal, etc. atraviesan una severa crisis económica, cuyos efectos son de alcance mundial, y que según los expertos internacionalistas refieren que sus estragos afectaran a todos los países del mundo, a unos con más intensidad que a otros, en el caso del Perú por contar con una economía social de mercado y con política de amplia apertura a las inversiones, le ha permitido generar las suficientes reservas monetarias que le dan estabilidad y solidez a nuestra economía, refieren que su impacto será moderado, permitiendo de esta forma impulsar los programas de inclusión social dentro una administración pública eficiente.

En este escenario mundial turbulento, nuestro país -para continuar con el desarrollo de sus sectores productivos y de servicios en el ámbito nacional y consolidar sus logros alcanzados en materia de la macroeconomía y proyectarse al crecimiento de la microeconomía en el corto plazo, tomando como base la inclusión socialdebería priorizar como objetivo estratégico y táctico fortalecer la gestión pública nacional con una propuesta que comprenda una reforma de la administración pública integral, que tome en cuenta la competitividad, calidad y la gestión del conocimiento como pilares fundamentales para su desarrollo y mejoramiento de los servicios que presta al país y así optimizar la gestión pública, mediante una revalorización del trabajador de la administración pública, con programas de profunda moralización en todos los niveles sectoriales y la implementación de una reingeniería de su organización con respecto a sus funciones, estructuras, cargos y procedimientos.

Los informes del FMI, BID y el BM precisan que la inversión pública y privada en nuestro país, debe centrar su atención en forma priorizada en 
los programas de inclusión social, no en una forma asistencialista sino a través del emprendimiento laboral con el objeto incrementar la población económicamente activa, indicador de la economía que refleja el crecimiento de la economía.

De igual forma, los programas de capacitación, perfeccionamiento y especialización de la fuerza laboral del sector público nacional requieren de la ejecución de herramientas modernas de desarrollo de personal, como son el coaching, mentoring $y$ consulting, que se constituirán en la palanca del desarrollo nacional, reflejándose en los programas de infraestructura de salud, educación y vial, en la elaboración de los proyectos de inversión económico y social, y en promover los convenios de cooperación técnica y económica nacional e internacional, que reactiven los sectores productivos y de servicios, mediante una gestión pública eficaz y eficiente, que posibilite ayudar a sostener la demanda interna y el empleo.

\section{GESTIÓN PÚBLICA EN EL PERÚ}

Los reportes macroeconómicos nacionales e internacionales referentes a nuestro país coinciden que la proyección de crecimiento de la economía es de $6.5 \%$ anual e insisten en que las fortalezas y oportunidades del Perú en el plano macroeconómico le permitirá capear la crisis económica mundial, en razón a la alta cotización del precio de los minerales, como es el caso del oro, plata, cobre, zinc, (exportación tradicional) y la agroindustria, como la exportación de los espárragos ( no tradicional), sumados a los efectos de los Tratados de Libre Comercio suscrito por el Perú con países desarrollados y en vías de desarrollo y fundamentalmente al consumo interno. En consecuencia, se hace necesario llevar a cabo una ambiciosa reforma estructural, que incluye la modernización de la administración pública del país.

Para garantizar el desarrollo de la economía en el Perú, es necesario que se mantenga la estabilidad en el sistema democrático, la alternancia del poder, la seguridad ciudadana, la independencia de los poderes del Estado, la libertad de prensa, la moralización en todos los niveles del sector público nacional, la administración de la justicia basada en el respeto en el ordenamiento jurídicolegal, contar con un sistema nacional de control transparente, etc. son los requisitos imprescindibles para garantizar una gestión pública eficaz, eficiente y efectiva.
Los partidos políticos en el Perú requieren de una modernización integral, deben responder a una filosofía propia o tener vínculos doctrinarios con partidos políticos internacionales y no responder a agrupaciones políticas que surgen de coyunturas internas de procesos electorales, donde aparecen personajes con mentalidad caudillista, totalitaria y con actitudes antidemocráticas, muchos de ellos con antecedentes penales, judiciales y policiales, situación que va en desmedro de la deontología profesional y a una pérdida de credibilidad por la sociedad peruana. El propósito fundamental de los partidos políticos es plantear planes de gobierno y planes de desarrollo hoy también llamados hojas de ruta, que responden a las demandas de los sectores productivos y sociales nacionales, dentro de los cuales debe considerarse la gestión pública y la inclusión social, como prioridades en la palanca de desarrollo del país.

Se debe promover e intensificar en el país, una imagen positiva de la gestión pública, que debe estar al servicio de la sociedad peruana, dentro de un contexto de excelencia y no ser considerado como un sistema burocrático, sobredimensionado y conflicto social. La elaboración de los planes de desarrollo nacional a corto, mediano y largo plazo permitirán que el sector empresarial privado plantee sus planes tácticos y estratégicos, no sólo basado en la investigación de mercados y en la rentabilidad empresarial, sino también en el interés nacional.

La tarea inmediata es generar una cultura organizacional propia para la gestión pública, basada en la ética, transparencia y eficiencia en su accionar y en la prestación de sus servicios, constituyéndose en un paradigma de desarrollo nacional, que responda a la siguiente interrogante:

¿Por qué es importante incorporar a los mejores trabajadores del país en la gestión pública nacional?

La experiencia demuestra que el éxito de la gestión pública comienza con el reclutamiento, selección, contratación e inducción de personas altamente calificadas para ocupar los puestos de trabajo, sean estos directivos, profesionales, técnicos, administrativos y de servicios, que acrediten una formación académica sólida y una experiencia laboral en la administración pública, para satisfacer los perfiles ocupacionales requeridos por los sectores productivos y sociales de la administración pública, dentro de un contexto de 
competencias laborales. Estas tecnologías modernas del talento humano serán la base del éxito y de la modernización de la administración pública.

Las competencias laborales del trabajador de la Administración Pública:

- Desarrollar su labor directiva, profesional, técnica, administrativa o de servicios, al más alto nivel del rendimiento laboral.

- Mejorar su calidad de vida en lo personal y en el trabajo.

- Trabajar con los mejores patrones o estándares de rendimiento individual y colectivo (equipo de trabajo).

- Demostrar sus capacidades, habilidades, destrezas y cualidades, y que está altamente preparado para enfrentar los retos de la modernidad de la gestión pública.

- Trabajar intensamente para alcanzar los objetivos y las metas institucionales.

- Ser proactivo en su accionar, tener fidelidad con la institución, vocación de servicio con él usuario y asumir un rol de liderazgo y competitividad laboral permanente.

- Desempeñarse en forma creativa, apostando por la innovación, resolviendo problemas en su trabajo.

- Aspirar a un sistema remunerativo justo, que le permita satisfacer sus necesidades.

Perfil del trabajador de la Administración Pública:

- Liderazgo y calidad en el cumplimiento de sus funciones.

- Adaptarse a los nuevos paradigmas del desarrollo institucional.

- Participación en la toma de decisiones en el ámbito de su competencia.

- Formar parte del trabajo en equipo, apostar por los consensos.

- Promover un clima motivador y de confianza en el trabajo.

- Desarrollo y capacitación del personal en forma permanente.

- Participar intensamente en los programas de Seguridad e Higiene Ocupacional y de Defensa Civil.
- Equilibrio familiar y laboral.

- Administrar los conflictos (inteligencia emocional).

Variables del perfil ocupacional del trabajador de la Administración Pública:

- Variables personales:

- Apostar por la vocación de servicio

- Lealtad para con su institución

- Fomentar la ética en su comportamiento laboral

- Desarrollar la autoestima personal y laboral

- Potenciar sus habilidades y destrezas

- Mejorar su rendimiento individual y en equipo para el cumplimiento de las funciones del puesto.

- Variables económicas:

- Remuneraciones justas y equitativas ajustadas a la realidad económica del país y a la calidad de vida del trabajador.

- Tener un sistema de evaluación por productividad, que incentive el rendimiento laborar.

- Mejorar las condiciones de los trabajadores.

CAPACITACIÓN PERMANENTE A LOS TRABAJADORES DE LA ADMINISTRACIÓN PÚBLICA

Lamentablemente, fue desactivada la Escuela Superior de Administración Pública (ESAP), perteneciente al entonces Instituto Nacional de la Administración Pública (INAP), institución formativa que desarrollaba una Maestría en Gestión Pública y Programas de Especialización para los sistemas de personal, abastecimiento y racionalización administrativa preferentemente, organismo rector de la administración en la que la tuve la responsabilidad de ser director nacional de racionalización y después director nacional de personal, situación que permite hacer una reflexión sobre lo que significa hacer abuso del poder, sin tomar en cuenta la importancia del rol que tienen estas entidades públicas en todos los países del mundo. No se puede prescindir de la capacitación, que es una inversión, las instituciones pueden ser conducidas en forma ineficiente, pero de ninguna manera pueden ser desactivadas, por el contrario debieron ser repotenciadas. Con 
trabajadores de la actividad pública capacitados permanentemente e idóneos, podremos involucrarnos en una profunda e irreversible reforma de la administración pública, debido que los trabajadores deben entrar con paso firme al camino del esfuerzo, la disciplina y la constancia y dejar atrás el consuelo de la mediocridad, burocracia y la ilusión de las cosas fáciles y de las prebendas.

La gestión pública eficiente requiere de una Escuela Superior de Administración Pública moderna con una infraestructura académica de tecnología de punta, con una planta docente que acredite gestión pública eficiente, equipamiento de laboratorios informáticos de última generación, etc., cuya filosofía central sea el mérito y el esfuerzo para enseñar a los trabajadores comprender la realidad nacional, regional y local, para tener capacidad de desarrollo y vocación de servicio.

El proyecto de mejorar nuestra sociedad y para impulsar el desarrollo del Perú es a través de la educación vía un proyecto educativo, que comprenda modernizar la gestión pública, sobre la base de que el ser humano es realmente libre solo con el conocimiento y la razón.

Sugerimos que la creación de la Escuela Superior Administración Pública del Perú tenga un rango universitario, donde se dará a los estudiantes lo mejor que puede ofrecerles el Estado y la sociedad y podrán acceder al título profesional de Licenciado en Administración Pública y a Programas de Maestría y Doctorado con menciones en Gestión Pública.

\section{LOS PROGRAMAS DE RESPONSABILIDAD SOCIAL EN LA ADMINISTRACIÓN PÚBLICA}

Es una experiencia que viene aplicándose en diversos sectores productivos y de servicios en el ámbito privado del país, como la minería, agroindustria y finanzas, entre otras, y debería aplicarse en el sector público nacional, su contribución es de manera activa y voluntaria, y su principal objetivo es optimizar la sociedad y la protección del medio ambiente.

Las empresas privadas consideran que los principios de los programas de responsabilidad social reflejan el nivel de aprobación, que pueden generar en la opinión pública sobre determinada actividad que desarrollan y asumen un compromiso con la sociedad para logran fidelizar a los clientes o usuarios, debido a que aumenta la imagen positiva que proyectan ante la comunidad, de igual modo este mismo comportamiento debe ser asumido por las entidades del Estado.

\section{Beneficio que se espera alcanzar}

Se espera mejorar la imagen de la organización pública frente a la comunidad nacional. Las organizaciones líderes que asumen estos programas son frecuentemente destacadas en los medios de comunicación social, y son recomendadas a los fondos de inversión.

Contribuyen con incrementar los niveles de lealtad de los empleados para con las instituciones públicas, debido a que promueven la estabilidad y el bienestar del público interno, también aseguran una buena relación con el entorno nacional e internacional, lo que les permite incrementar la influencia en la sociedad.

De este modo, publicar información honesta, precisa y emprender acciones socialmente comprometidas, mejora la imagen de la entidad pública frente a la sociedad civil y genera nexos que puedan desencadenar en proyectos sociales.

\section{Menos costo:}

- No solo las empresas privadas con gran capital pueden ser socialmente responsables.

- La Gestión Pública también pueden comprometerse con la comunidad y obtener los beneficios de involucrarse con la sociedad nacional.

Una entidad pública que destine recursos en realizar acciones sociales le otorgará a sus productos o servicios valor agregado, de igual forma si se destina parte de su presupuesto en acciones sociales o ambientales tiene una actuación superior de aquellas que no lo desarrollan. Además las prácticas sociales filantrópicas obtienen tasas de retorno a sus inversiones muy superiores a sus expectativas.

\section{EL TALENTO HUMANO EN LA ADMINISTRACIÓN PÚBLICA}

Uno de los factores de éxito o de fracaso en las instituciones públicas descansa en buena parte en la calidad e idoneidad del potencial humano incorporado, que se deriva del conjunto de políticas, procedimientos e instrumentos usados para su identificación, reclutamiento, selección, contratación, vinculación, orientación y dirección necesarios para la realización de las funciones asignadas. 
El punto de partida de las políticas de empleo y desarrollo de personal es el nombramiento o contratación, según sea el caso y la asunción de funciones, tareas y responsabilidades por parte del trabajador, dentro de un contexto de competencias laborales.

Por tanto, el proceso de convocatoria, selección y contratación suele ser riguroso, pues se busca un trabajador con las mejores calidades humanas, experiencia laboral, formación académica acreditada, ética, madurez, carácter y, además, de una comprobada capacidad de adaptación a los retos propios de cualquier proyecto de alguna magnitud.

Estos comentarios responden a mi experiencia en la gestión pública por más de 30 años en diversas instituciones del Estado, en cargos de diferentes niveles de responsabilidad; a mi criterio, son simples aportes que requieren ser enrriquecidos.

\section{LITERATURA CITADA}

KLINKSBERG, Bernardo. ¿Cómo transformar al Estado?. Más allá de mitos y dogmas. Santiago de Chile, 1989.
LOZADA Y MORRADAN, Carlos. De Burócratas a Gerentes. Banco Interamericano de Desarrollo, Washington, 1999.

MATOS MAR, José. Desborde Popular y Crisis del Estado. IEP. Lima, 1986.

MILLA BATRES, editores. En que momento se jodió el Perú. Editorial Milla. Lima, 1990.

PINEDA, Juan de Dios. Enfoques Contemporáneos de la Administración Pública. Ciudad de México, 2000.

PATRON FAURA, Pedro; PATRON BEDOYA, Pedro, Derecho administrativo y Administración Pública en el Perú. Lima: Grijley, 2004

"La especificidad de la gestión pública" en De burócratas a gerentes (BID cap. 1 Echevarría y Mendoza.

"Liderazgos, políticas y gestión pública para la nueva gobernabilidad" en Democracia en déficit (BID Cap. VIII). Borbón, José Octavio. 